\title{
Modelling tensile force oscillation during the tensile test of PET specimens
}

\author{
L. M. Vas, F. Ronkay*, T. Czigány \\ Department of Polymer Engineering, Faculty of Mechanical Engineering, Budapest University of Technology and \\ Economics, Múegyetem rkp. 3. Budapest H-1111, Hungary \\ Received 30 October 2008; accepted in revised form 13 December 2008
}

\begin{abstract}
Force oscillation occurring during the tensile testing of poly(ethylene terephthalate) (PET), resulting in periodical cavitation of the test specimens, has been studied. A mathematical model has been developed to describe the phenomenon, wherein special fibre bundles are assigned to the amorphous molecular chains. In order to model the local periodical transformations and the rate dependent viscoelastic behaviour the coupled fibre bundle cells were supplemented with a twoelement Maxwell model. Using the parameters determined from the measurements the model was compared to the measured force-elongation diagrams and it has been concluded that the simple model can be well used to describe the phenomenon.
\end{abstract}

Keywords: modelling and simulation, stress oscillation, viscoelastic properties

\section{Introduction}

The viscoelastic properties of the polymers have been widely studied [1]. Recently its modelling also came to the front [2]. Stress oscillation occurring during the tensile testing of poly(ethylene terephthalate) (PET) - also denoted as self-oscillation - was described first in 1970, in conjunction with necking induced by the static loading of amorphous PET [3]. Stress oscillation means that under certain conditions the stress arising during necking is not constant anymore, but exhibits a periodic fluctuation vs. time [4]. The reasons of stress oscillation have not been clarified in the literature and are hotly debated [5]. Among possible explanations one can find local heating caused by orientational elongation [6-10]; oscillation of local deformation rate during necking within a critical stress range [11]; orientation crystallization induced by adiabatic heat formation [12].
Karger-Kocsis et al. $[13,14]$ studied the oscillation phenomenon in sPP. In their opinion necking, which is a prerequisite of stress oscillation in any polymer, produces an elongated, random network. This network closely resembles partially crystalline thermoplastic elastomers. The deformation of this complex system is strongly inhomogeneous. This, by itself, induces a shear deformation process. According to their explanation, when some small crystallites disintegrate during the tensile elongation the shear deformation is amplified further and cavities are formed at the crossing of shear bands. If the density of micro-waves of crossings reaches a critical value the material is weakened and its loadbearing capacity drops because of the abrupt cavitation. This explains the stress drop in the amplitude of stress oscillation. According to this explanation shear bands cannot easily cross each other on the sample surface, which is in agreement with

*Corresponding author, e-mail: ronkay@pt.bme.hu

(C) BME-PT and GTE 
the experimental observation that cavities always appear inside the test specimen.

According to Ebener et al. [15] oscillation arises if the test specimen can store enough elastic energy. For this the initial length of the test specimen should be large enough. Elastic energy storagecapacity can also be achieved by the application of an external spring. According to his measurements, if the deformation rate is increased during the oscillation, the mean stress and the amplitude decrease, while local heat removal results in the increase of the mean stress. In his opinion during the deformation - a high degree of orientation occurs and heat is released - the polymer may crystallize partially in both the transparent and non-transparent parts. As the local deformation rate is higher during the formation of the non-transparent bands, crystallization is easier here. Not all non-transparent bands were found, however, to be partially crystalline.

In our earlier study [16] we traced back the origin of the oscillation to the cavitation of the test specimen. We observed that the cavitation occurs simultaneously with the stress oscillation, in a periodic manner, before reaching the stress peaks. Due to the formation of the cavities the heat conductivity of the material decreases considerably, the temperature of the deformation zone increases abruptly (with $36-40^{\circ} \mathrm{C}$ ), resulting in a drop of the strength and in elongation. During neck propagation, however, the material cools down, the strength increases again, so the process becomes periodical. Our conclusions were corroborated by SEM (scanning electron microscopy) and $\mathrm{AE}$ (acoustic emission) measurements. In order to describe the phenomenon a physical model was constructed, which traces back the phenomenon to changes of the molecular structure. According to this model shear forces arise between the molecular chains orienting under the effect of stress and, as a result, the material splits into molecular bundles. Ordering of the molecules into bundles is accompanied by significant crystallization. Micro-cracks (crazes) between the bundles decrease the heat conductivity of the material considerably and this leads to local heating.

In this article the changes in the orientation of the molecular chains are modelled by fibre bundles. Fibres of a bundle consisting of partially ordered, homogeneous (uniform) fibres can be classified according to their initial state and according to the properties of their environment. Fibres belonging to the same class form a partial bundle, a so-called fibre bundle cell. In this article a pre-stressed, breaking fibre bundle (so-called EH bundle) is used $[17,18]$, with independent fibres, the strings defined by the clamping points are well ordered, but the individual fibres may be either pre-stressed with a statistically variable $\varepsilon_{0}$ elongation $\left(\varepsilon_{0}>0\right)$, or may be loose, i.e. crimped $\left(-1<\varepsilon_{0}<0\right)$. In order to describe the viscoelastic behaviour of the test specimen a two-element Maxwell model is used [19].

The main goal of this study is to develop a simple mathematical model to describe the force-elongation curve. Aim was to model two phenomena known and explained: 1. Oscillation occurs just over a strain rate; 2 . Oscillation is connected with forming crazes where amorphous molecular chains like fibres get stressed/strained and create bundles of chains and voids among them.

\section{Experimental results}

\subsection{Material and equipment}

In our experiments standard 'dumb-bell' specimens were injection molded from PET (SkyPET-BL 8050, SK Chemicals) pellets using an Arburg Allrounder $270 \mathrm{C}$ injection moulding machine. Zone temperatures of the injection moulding machine were: $235 / 240 / 245 / 250 / 255^{\circ} \mathrm{C}$, injection pressure was 500 bar. The mould was cooled by $15^{\circ} \mathrm{C}$ water. Tensile testing of the specimens was performed by a Zwick Z020 universal tensile tester at room temperature. Cavities and crazing appearing in the material were studied by a JEOL JSM 6380LA electron microscope.

\subsection{Measurements}

Tensile tests were performed at a tensile rate of $60 \mathrm{~mm} / \mathrm{min}$, the initial gauge length of the test specimens was $115 \mathrm{~mm}$. Tensile curve shown in Figure 1 was obtained during the test. As shown by the figure, after a uniform neck propagation an oscillation of about constant amplitude (5.8 MPa) and cycle time (1.14 s) appears in the stress. The lower limiting value of the oscillation is $21.2 \mathrm{MPa}$, its upper limiting value is $27 \mathrm{MPa}$. The slope of the 


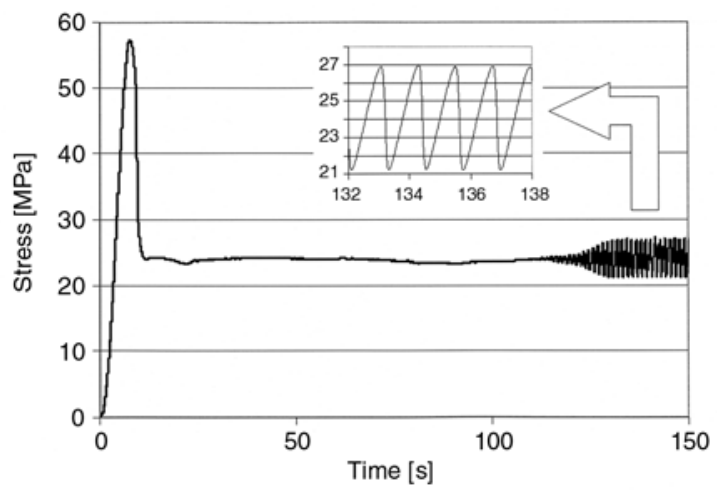

Figure 1. Tensile curve of PET at a tensile rate of $60 \mathrm{~mm} / \mathrm{s}$

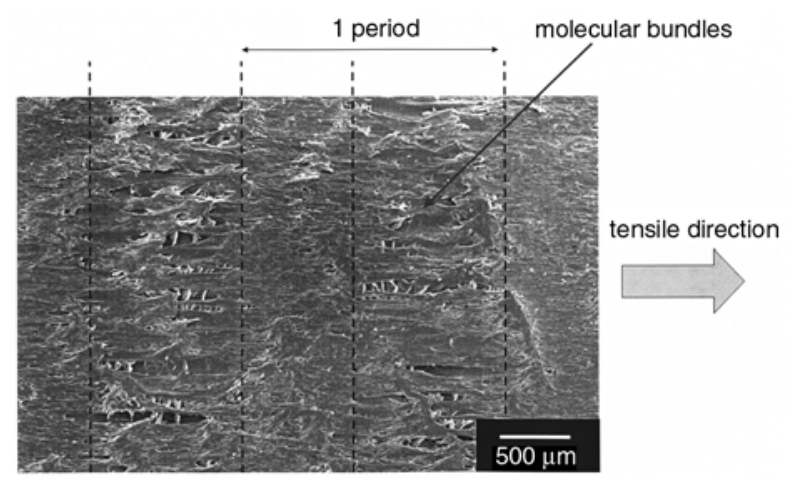

Figure 2. SEM micrograph of the longitudinal section of the specimen

tangent at the initial portion of the curve is $4.5 \mathrm{MPa} / \mathrm{s}$, in the initial portions of the oscillating period it is, however, $6 \mathrm{MPa} / \mathrm{s}$.

Figure 2 shows the longitudinal section of a specimen which has undergone oscillation. One can see that the cavitation occurs periodically. Between the cavities parallel with the load molecular bundles can be observed with a thickness between 10 and $150 \mu \mathrm{m}$. The bands perpendicular to the tensile elongation without cavities are formed during the increasing stress interval of the stress oscillation period, while the bands containing cavities are formed during the stress drop interval.

\section{Modelling the force oscillation and discussion}

Based on the tensile tests of PET specimens the force oscillation appears only after the initiation of necking, above a certain tensile rate, indicating a load peak, therefore it can be characterized by a critical stress $\left(\sigma_{\text {crit }}\right)$. When neglecting the initial load peak the force-elongation curve increases monotonously. Two forms of this is shown in Fig-

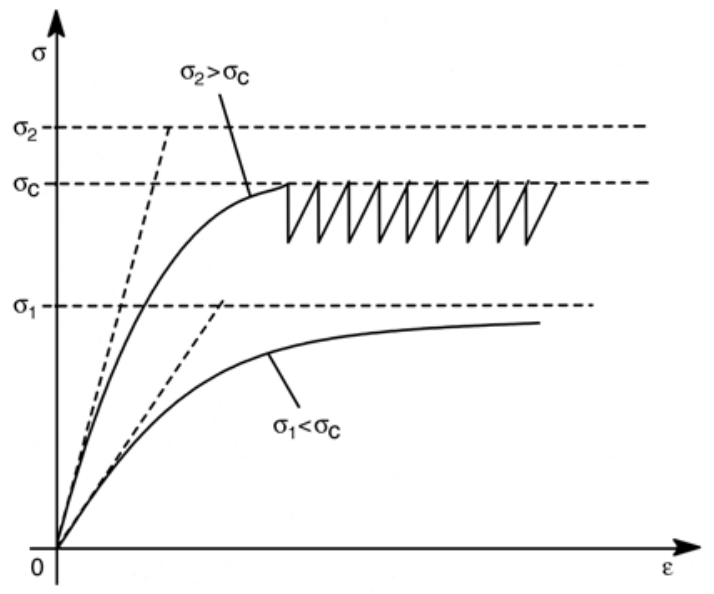

Figure 3. Force responses to be modelled

ure 3 . In one case the asymptotic stress $\left(\sigma_{1}\right)$ is less than $\sigma_{c}=\sigma_{c r i t}$ therefore oscillation does not take place. In the other case this stress $\left(\sigma_{2}\right)$ is exceed $\sigma_{\text {crit }}$ hence reaching $\sigma_{\text {crit }}$ the oscillation starts.

Based on these an analogous mechanical model was developed in order to describe mathematically the characteristics and the force oscillation shown in Figure 1 and to study the phenomenon analytically.

\subsection{Mechanical model}

As shown by the experiments crazing and void formation occurs periodically. It means that the molecular chains in the amorphous phase assemble into micro-bundles in front of the deformation zones forming voids, some of which may develop into macro-cavities. Amorphous chains can be considered as part of an EH type fibre bundle $[17,18]$ consisting of crimped fibres which are perfectly flexible and do not transmit tensile force while being crimped (Figure 4).

It is assumed that the EH bundle cell is enclosed into a rigid box which remains intact until exposed to a critical force level. Then the box opens and extends telescopically, but a force is transmitted only when the fibres are straightened (see Figure 4). For the sake of simplicity let us consider the
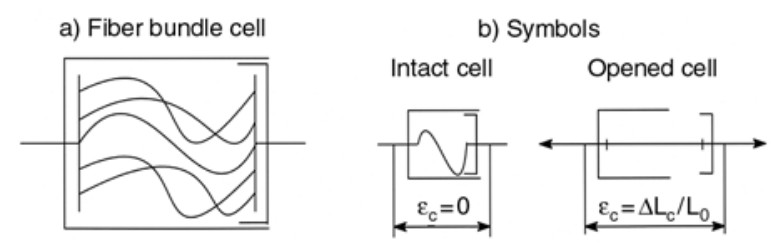

Figure 4. Fiber bundle cell and its symbols in intact and opened states 


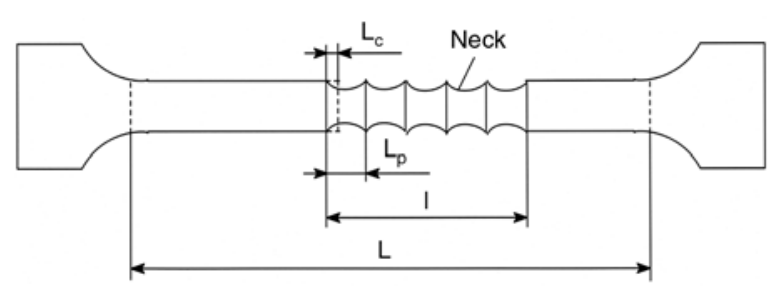

Figure 5. Specimen with neck periods

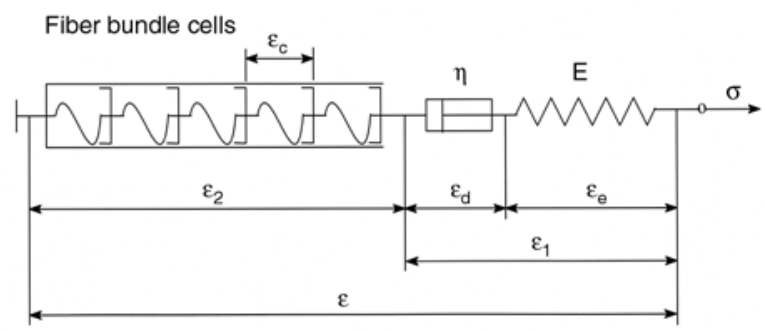

Figure 6. Analogous mechanical model

fibres as inextensible, the boxes as rigid and the level of crimping uniform. In this case the deformation of the intact cell is zero, while that of the opened cell is described in Equation (1):

$\varepsilon_{c}=\frac{\Delta L_{c}}{L_{0}}=\frac{L_{p}-L_{c}}{L_{0}}=\frac{L_{p}-L_{p}\left(1+\varepsilon_{f_{0}}\right)}{L_{0}}=-\varepsilon_{f_{0}} \frac{L_{p}}{L_{0}}$

where $L_{0}$ is the gauge length of the test specimen, $L_{c}$ and $L_{p}$ are the lengths of the intact and open cells respectively, $\varepsilon_{f_{0}}<0$ is the crimping of the fibres (see Figure 5). Note that $L_{p}$ is a kind of period length measurable on the neck.

In order to model the periodical local transitions and the viscoelastic rate-dependent behaviour of the specimen a stiff box containing a number of serially connected fibre bundle cells and a two-element Maxwell model [19] are used, respectively (see Figure 6).

\subsection{Force oscillation response}

Modelling the tensile test the constant rate elongation of the model shown in Figure 6 is described with Equation (2):

$$
u(t)=\varepsilon(t)=\frac{L-L_{0}}{L_{0}}=\frac{\Delta L}{L_{0}}=\frac{v t}{L_{0}}=\dot{\varepsilon}_{0} t
$$

where $u$ is the relative extension of the specimen measured between the grips and $\dot{\varepsilon}_{0}$ is the strain rate. The response of the model changes in time intervals because the fibre cells open one after another periodically causing jump-like drops in the force. The strain rate is equal to $\dot{u}$, the rate of extension of the specimen measured between the grips, as described in Equation (3):

$\dot{u}=\dot{\varepsilon}_{0}=\frac{v}{L_{0}}$

where $v$ and $L_{0}$ are the speed of the moving grip and the gauge length of the specimen, respectively.

In the first time interval up to the first load peak $\left(0 \leq t \leq t_{1}\right)$ every cell is closed, hence only the spring $\left(E=E_{0}\right)$ and the dashpot $\left(\eta=\eta_{0}\right)$ of the Maxwell model deform (Figure 6: $\varepsilon_{2}=0, \varepsilon=\varepsilon_{1}$ ), which can be described by Equation (4):

$$
\dot{\varepsilon}=\frac{\dot{\sigma}}{E_{0}}+\frac{\sigma}{\eta_{0}}
$$

The general solution of Equation (4) for a stimulus according to Equation (2) can be derived easily in Equation (5):

$$
\begin{gathered}
\sigma(t)=\sigma(0) e^{-t / \tau_{0}}+E_{0} \dot{\varepsilon}_{0} \tau_{0}\left(1-e^{-t / \tau_{0}}\right) \underset{t \rightarrow \infty}{\longrightarrow} \\
E_{0} \dot{\varepsilon}_{0} \tau_{0}=\eta_{0} \dot{\varepsilon}_{0}=\sigma_{\text {Lim }}
\end{gathered}
$$

which tends to a constant stress value $\left(\sigma_{\text {Lim }}\right)$, where $\tau_{0}$ is a time constant, which is described by Equation (6):

$$
\tau_{0}=\frac{\eta_{0}}{E_{0}}
$$

Supposing that $\sigma(0)=0$, the stress-strain relationship for the first interval $\left(0 \leq t \leq t_{1}\right)$ is given by Equation (7):

$$
\sigma(t)=E_{0} \dot{\varepsilon}_{0} \tau_{0}\left(1-e^{-t / \tau_{0}}\right)
$$

The initial slope in this interval depends on the strain rate and is described by Equation (8):

$$
\dot{\sigma}(0)=E_{0} \dot{\varepsilon}_{0}=\dot{\sigma}_{0}
$$

Consequently, increasing the strain rate increases both the initial slope $\left(\dot{\sigma}_{0}\right)$ and the asymptotic stress value $\left(\sigma_{\text {Lim }}\right)$, therefore, if the strain rate is large enough the stress reaches the critical stress in a finite time $\left(0<t_{1}<\infty\right)$ (Equation (9)):

$\sigma\left(t_{1}\right)=\sigma_{c r i t}$ 


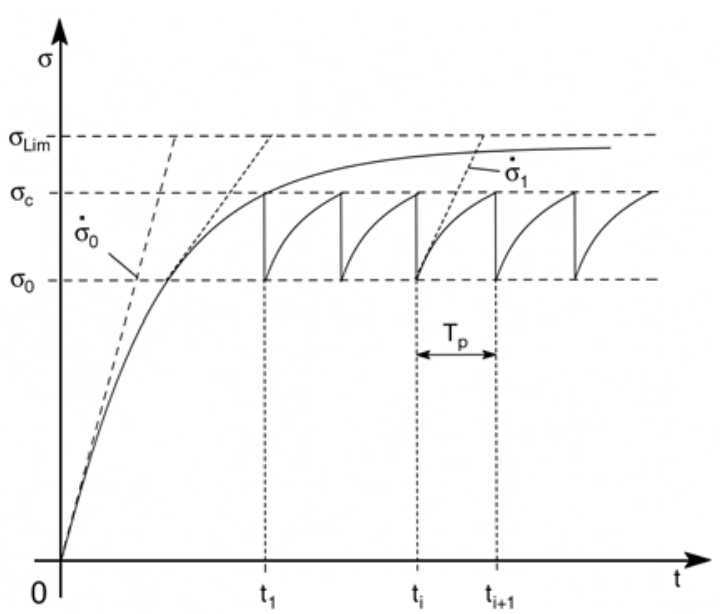

Figure 7. Model response

Reaching the critical stress at time point $t_{1}$ the first fibre cell opens and the strain of the spring decreases suddenly by $\varepsilon_{c}$, up to the entire straightening of the fibres $\left(\varepsilon_{e}\left(t_{1}\right)>\varepsilon_{c}\right)$, and the stress drops to $\sigma_{0}$ (Figure 7). In the real PET material a neck forms at the first force peak, where the extension is cumulated, the strain becomes essentially localized, hence the strain rate increases: $\dot{\varepsilon}_{1}>\dot{\varepsilon}_{0}$. Hence, if the length of the neck formed at $t_{1}$ is $l_{1}$, and assuming that the local extension $(\Delta l)$ in the $\left(t_{1}, t_{1}+\Delta t\right)$ interval is equal to that of the specimen or the entire model $\left(\Delta L_{1}\right)$, i.e. if the extension of the propagating neck outside of the given section is negligible, which is described in Equation (10):

$$
\Delta l=l-l_{1}=\Delta L_{1}=L-L_{1}=v \Delta t
$$

where $L_{1}=L\left(t_{1}\right)$. Accordingly the local strain and strain rate are (see Figure 6) described in Equations (11) and (12):

$$
\begin{aligned}
& \Delta \varepsilon_{1}=\frac{\Delta l}{l_{1}}=\frac{v}{l_{1}} \Delta t=\dot{\varepsilon}_{1} \Delta t \\
& \dot{\varepsilon}_{1}=\frac{v}{l_{1}}=\frac{v}{L_{0}} \frac{L_{0}}{l_{1}}=\dot{\varepsilon}_{0} \frac{L_{0}}{l_{1}}>\dot{\varepsilon}_{0}
\end{aligned}
$$

As another consequence a kind of hardening takes place because of the increase in the orientation. Therefore, in an analogous way, the model parameters are supposed to change as well: $E=E_{1}$ and $\eta=\eta_{1}$.

On the basis of the considerations above Equation (13) is valid for the second time interval $\left(t_{1}<t \leq t_{2}\right)$ :

$$
\dot{\varepsilon}_{1}=\frac{\dot{\sigma}}{E_{1}}+\frac{\sigma}{\eta_{1}}
$$

The general solution of Equation (13) is in Equation (14), which is similar to that of Equation (5):

$$
\begin{gathered}
\sigma(t)=\sigma\left(t_{1}+0\right) e^{-\left(t-t_{1}\right) / \tau_{1}}+E_{1} \dot{\varepsilon}_{1} \tau_{1}\left(1-e^{-\left(t-t_{1}\right) / \tau_{1}}\right) \underset{t \rightarrow \infty}{\longrightarrow} \\
E_{1} \dot{\varepsilon}_{1} \tau_{1}=\eta_{1} \dot{\varepsilon}_{1}=\sigma_{\text {Lim }_{1}}
\end{gathered}
$$

where $\tau_{1}$ is a new time constant (Equation (15)):

$$
\tau_{1}=\frac{\eta_{1}}{E_{1}}
$$

Here $\sigma\left(t_{1}+0\right)=\sigma_{0}$, hence the stress-strain relationship and the initial slope for the second interval $\left(t_{1}<t \leq t_{2}\right)$ are given by Figure 7 and it is described in Equations (16) and (17):

$$
\begin{aligned}
& \sigma(t)=\sigma_{0} e^{-\left(t-t_{1}\right) / \tau_{1}}+E_{1} \dot{\varepsilon}_{1} \tau_{1}\left(1-e^{-\left(t-t_{1}\right) / \tau_{1}}\right) \\
& \dot{\sigma}\left(t_{1}+0\right)=E_{1} \dot{\varepsilon}_{1}-\frac{\sigma_{0}}{\tau_{1}}=\dot{\sigma}_{1}
\end{aligned}
$$

It can be assumed that the phenomena in the next and further intervals are similar to those in the second interval, consequently the force process is periodical.

\subsection{Method of determining the model parameters}

Pre-set data of the tensile test are $L_{0}$ and $v$. From measurements performed on the PET specimens the following data can be determined directly: $\sigma_{c r i t}$, $\sigma_{0}, \dot{\sigma}_{0}, \dot{\sigma}_{1}, \dot{\varepsilon}_{0}, \dot{\varepsilon}_{1}, l_{0}, t_{1}, \ldots, t_{n}$, and $T_{p}$ (see Table 1).

Model parameters, such as $E_{0}, E_{1}, \eta_{0}, \eta_{1}, \varepsilon_{c}$ and $\varepsilon_{f_{0}}$ can be calculated from Equation (1-17).

If $\sigma_{0}$ is known $E_{0}$ can be determined from Equation (8) and solving the Equation (18) $\tau_{0}$ can be obtained:

$$
\sigma_{c r i t}=E_{0} \dot{\varepsilon}_{0} \tau_{0}\left(1-e^{-t_{1} / \tau_{0}}\right)
$$

and from Equation (6) $\eta_{0}$ can be calculated.

Equation (19) is given by Equation (16) for the $(i+1)$-th time interval at $t_{i+1}$ : 
Table 1. Test results

\begin{tabular}{|c|c|c|c|c|c|c|}
\hline$v$ & $L_{0}$ & $\sigma_{\text {crit }}$ & $\sigma_{0}$ & $\dot{\sigma}_{0}$ & $\dot{\sigma}_{1}$ & $\sigma_{\text {lim }}$ \\
\hline$[\mathrm{mm} / \mathrm{min}]$ & {$[\mathrm{mm}]$} & {$[\mathrm{MPa}]$} & {$[\mathrm{MPa}]$} & {$[\mathrm{MPa} / \mathrm{s}]$} & {$[\mathrm{MPa} / \mathrm{s}]$} & {$[\mathrm{MPa}]$} \\
\hline 60 & 115 & 27 & 21.1 & 4.5 & 7.2 & 36.3 \\
\hline$\dot{\varepsilon}_{0}$ & $\dot{\varepsilon}_{1}$ & $l_{1}$ & $L_{c}$ & $L_{p}$ & $t_{1}$ & $T_{p}$ \\
\hline$[1 / \mathrm{s}]$ & {$[1 / \mathrm{s}]$} & {$[\mathrm{mm}]$} & {$[\mathrm{mm}]$} & {$[\mathrm{mm}]$} & {$[\mathrm{s}]$} & {$[\mathrm{s}]$} \\
\hline 0.01 & 0.04 & 26 & 0.02 & 1.32 & 9.24 & 1.14 \\
\hline
\end{tabular}

$$
\sigma_{c r i t}=\sigma_{0} e^{-\left(t_{i+1}-t_{1}\right) / \tau_{1}}+E_{1} \dot{\varepsilon}_{1} \tau_{1}\left(-e^{-\left(t_{i+1}-t_{1}\right) / \tau_{1}}\right)
$$

Equation (20) is given by expressing $E_{1}$ form Equation (17) and substituting into Equation (16) and taking into consideration that $T_{p}=t_{i+1}-t_{i}$ :

$$
\sigma_{\text {crit }}=\sigma_{0} e^{-T_{p} / \tau_{1}}+\left(\dot{\sigma}_{1} \tau_{1}+\sigma_{0}\right)\left(1-e^{-T_{p} / \tau_{1}}\right)
$$

Solving Equation (20) $\tau_{1}$ is obtained, then from Equation (15) and (17) $\eta_{1}$ and $E_{1}$ can be calculated respectively. Finally, the negative strain $\left(\varepsilon_{c}\right)$ stored as crimping or loosening in the fiber cells can be calculated in the Equation (21):

$\sigma_{c r i t}-\sigma_{0}=E_{1} \varepsilon_{c} \rightarrow \varepsilon_{c}=\frac{\sigma_{c r i t}-\sigma_{0}}{E_{1}}$

The length of the $L_{p}$ period can be measured on the neck, therefore the length of the intact cell $\left(L_{c}\right)$ and the fibre crimping $\left(\varepsilon_{f_{0}}\right)$, as a negative strain can be determined from Equations (22) and (23):

$$
\begin{aligned}
& L_{c}=L_{p}-\varepsilon_{c} L_{0} \\
& \varepsilon_{f_{0}}=-\varepsilon_{c} \frac{L_{0}}{L_{p}}
\end{aligned}
$$

As a cross-check it is worth to calculate the cycle time from Equation (20) and to compare it with the measured value in Equation (24):

$$
\begin{gathered}
T_{p}=t_{i+1}-t_{i}=\tau_{1} \ln \frac{\eta_{1} \dot{\varepsilon}_{1}-\sigma_{0}}{\eta_{1} \dot{\varepsilon}_{1}-\sigma_{c r i t}}= \\
\tau_{1} \ln \frac{\dot{\sigma}_{1} \tau_{1}}{\dot{\sigma}_{1} \tau_{1}+\sigma_{0}-\sigma_{c r i t}}
\end{gathered}
$$

Substituting the model parameters calculated from the measured data (Table 2) into the equations above the oscillation curve predicted by the model is obtained.
Table 2. Parameters obtained by model fitting

\begin{tabular}{|c|c|c|c|c|c|c|c|}
\hline$E_{0}$ & $E_{1}$ & $\eta_{0}$ & $\eta_{1}$ & $\varepsilon_{c}$ & $\varepsilon_{f_{0}}$ & $\tau_{0}$ & $\tau_{1}$ \\
\hline$[\mathrm{MPa}]$ & {$[\mathrm{MPa}]$} & {$[\mathrm{MPas}]$} & {$[\mathrm{MPas}]$} & {$[-]$} & {$[-]$} & {$[\mathrm{s}]$} & {$[\mathrm{s}]$} \\
\hline 483.0 & 523.8 & 4174.5 & 853.7 & 0.01 & -0.98 & 8.64 & 1.63 \\
\hline
\end{tabular}

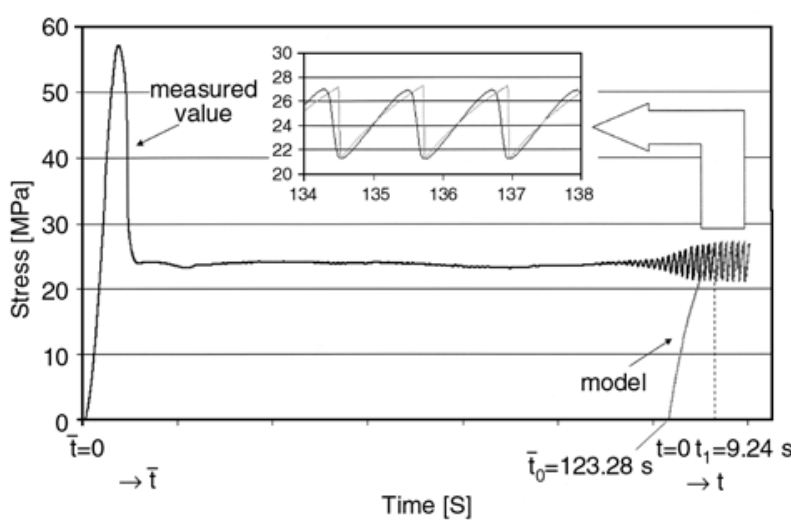

Figure 8. Comparison of the measured values and the model

Figure 8 compares the measured tensile curve with the points predicted by the model. As the model describes only the established oscillation, a better picture is obtained if the curve starts not in the true $t=0$ origin, but only immediately before the onset of the oscillation. As shown by Figure 8 the amplitude and frequency of the model curve are exactly identical with the corresponding values of the observed stress oscillation. The shape disagreement between the two curves is due to the fact that in the fibre bundle cells in the simplified model contain only idealized, inextensible fibres of uniform crimping, not real fibre bundles containing fibres of variable crimping, which would round off the sharp peaks of the curves.

\section{Conclusions}

Stress oscillation occurring during the tensile test of PET has been studied and modelled. A mathematical model was created to describe the oscillation. The amorphous molecular chains were described as apart of modelling by special EH-type fibre bundles containing crimped fibres enclosed into rigid boxes. These boxes open up at a certain critical force value, when the crimped fibres straighten and begin to bear the load. In order to model the periodic local transformation and the rate-dependent viscoelastic behaviours of the test specimens the connected fibre bundle cells were supplemented by a two-element Maxwell model. 
Using the parameters determined from the measurements the model was compared to the real tensile curve and concluded that simple model can be well used to describe the phenomenon.

\section{Acknowledgements}

This work has been supported by the Hungarian Scientific Research Fund through grants OTKA NI 62729, OTKA K049069 and PD72722. The author of this paper is a grantee of the Bolyai János Scholarship.

\section{References}

[1] Nagy P., Vas L. M.: Relationship between constant strain rate and stress relaxation behavior of polypropylene. Express Polymer Letters, 1, 84-91 (2007). DOI: $10.3144 /$ expresspolymlett.2007.15

[2] Felhős D., Xu D., Schlarb A. K., Váradi K., Goda T.: Viscoelastic characterization of an EPDM rubber and finite element simulation of its dry rolling friction. Express Polymer Letters, 2, 157-164 (2008). DOI: $10.3144 /$ expresspolymlett.2008.21

[3] Kechek'yan A. S., Adrinova G. P., Kargin V. A.: Periodic oscillation during stretching of polyethyleneterephthalate. Polymer Science U.S.S.R., 12, 2743-2757 (1970).

[4] Karger-Kocsis J., Benevolenski O. I., Moskala E. J.: Toward understanding the stress oscillation phenomenon in polymers due to tensile impact loading. Journal of Materials Science, 36, 3365-3371 (2001). DOI: 10.1023/A:1017935323058

[5] Karger-Kocsis J.: Fracture and fatigue behavior of amorphous (co)polyesters as a function of molecular and network variables. in 'Handbook of thermoplastic polyesters' (ed.: Fakirov S.) Vol1, Wiley-VHC, Weinheim, 734-756 (2002).

DOI: $10.1002 / 3527601961 . c h 16 a$

[6] Barenblatt G. I.: Self-oscillating neck propagation in polymers. Bulletin of the Russian Academy of Sciences: Mechanics of Solids, 5, 110-118 (1970).

[7] Adrinova G. P., Kechekyan A. S., Kargin V. A.: Selfoscillation mechanism of necking on extension of polymers. Journal of Polymer Science, Part A-2: Polymer Physics, 9, 1919-1933 (1971).

DOI: $10.1002 /$ pol.1971.160091101

[8] Roseen R.: Temperature effect at self-oscillating necking during extension of polyethylene terephthalate (PETP). Journal of Materials Science, 9, 929-933 (1974). DOI: $10.1007 / \mathrm{BF} 00570385$
[9] Toda A.: Oscillation and instability of neck propagation in poly(ethylene terephthalate) films. Polymer, 34, 2306-2314 (1993).

DOI: $10.1016 / 0032-3861(93) 90813-\mathrm{P}$

[10] Toda A.: Oscillatory neck propagation in polymerfilms. 2. Polymer, 35, 3638-3642 (1994). DOI: $10.1016 / 0032-3861(94) 90540-1$

[11] Pakula T., Fischer E. W.: Instabilities of the deformation process during cold drawing of poly(ethylene terephthalate) and other polymers. Journal of Polymer Science: Polymer Physics Edition, 19, 1705-1726 (1981). DOI: $10.1002 /$ pol.1981.180191104

[12] Godovsky Y. K.: Thermodynamic behavior of solid polymer in plastic deformation and cold drawing. in 'Thermophysical properties of polymers' (ed.: Godovsky Y. K. ) Springer-Verlag, Berlin, 221-229 (1992).

[13] Karger-Kocsis J., Shang P. P.: A modulated DSC study on the stress oscillation phenomenon in a syndiotactic polypropylene. Journal of Thermal Analysis and Calorimetry, 69, 499-507 (2002).

DOI: $10.1023 / \mathrm{A}: 1019903605228$

[14] Gutierrez M. C. G., Karger-Kocsis J., Riekel C.: Stress oscillation-induced modulated phase transformation and yielding in syndiotactic polypropylene. Chemical Physics Letters, 398, 6-10 (2004). DOI: $10.1016 /$ j.cplett.2004.09.023

[15] Ebener H., Pleuger B., Petermann J.: Stress and strain oscillations in syndiotactic polypropylene and in poly(ethyleneterephthalate). Journal of Applied Polymer Science, 71, 813-817 (1999).

DOI: $10.1002 /($ SICI $) 1097-$ 4628(19990131)71:5<813::AID-APP15>3.0.CO;2-P

[16] Ronkay F., Czigány T.: Cavity formation and stressoscillation during the tensile test of injection molded specimens made of PET. Polymer Bulletin, 57, 989-998 (2006). DOI: $10.1007 / \mathrm{s} 00289-006-0670-8$

[17] Vas L. M., Császi F.: Use of composite-bundle theory to predict tensile properties of yarns. Journal of the Textile Institute, 84, 448-463 (1993). DOI: $10.1080 / 00405009308658977$

[18] Vas L. M., Rácz Zs.: Modeling and testing the fracture process of impregnated carbon fiber roving specimens during bending part I. Fiber bundle model. Journal of Composite Materials, 38, 1757-1785 (2004). DOI: $10.1177 / 0021998304044767$

[19] Aklonis J. J., MacKnight W. J., Shen M.: Introduction to polymer viscoelasticity. Wiley-Interscience, New York (1972). 